\title{
Adaptação de um modelo lógico da atenção à Hipertensão Arterial na Atenção Básica: uma Revisão Sistemática
}

\author{
Adaptation of a logical model of attention to Hypertension In Primary Care: a \\ Systematic Review.
}

\begin{abstract}
Liane Batista da Cruz Soares ${ }^{1}$, Ivan de Abreu Figueiredo ${ }^{1,2}$
Resumo: $O$ presente estudo tem como objetivo realizar uma revisão sistemática sobre 0 modelo lógico da atenção à hipertensão arterial na atenção básica. A revisão sistemática foi realizada em setembro de 2014, em 3 bases de dados indexadas: Periódicos CAPES, SciELO e MEDLINE. Foi identificado um artigo que descreveu a construção de um Modelo Lógico. Após análise do modelo lógico proposto e dos documentos oficiais do Ministério da Saúde, foram realizadas algumas modificações no componente de prevenção, componente de cuidado, processo (retirou-se orientação sobre hábitos e estilos de vida saudáveis e programação de atividade física; acrescentaram-se grupos terapêuticos de hipertensos, educação continuada para hipertensos sobre alimentação saudável e incentivo a atividade física). No componente controle, componente de cuidado, processo (acrescentou-se estratificação). Espera-se que o modelo lógico proposto sirva de "modelo" para as ESF, a fim de que ocorram mudanças decorrentes dos produtos gerados pelas ações. Mudanças especificas no comportamento, conhecimento, habilidades, ou nível de desempenho do participante do programa, que poderão incluir melhoria da qualidade de vida.
\end{abstract}

Palavras-chave: Modelo Lógico. Hipertensão. Estratégia Saúde da Família.

\begin{abstract}
This study aims to conduct a systematic review of the logical model of healthcare for hypertension in primary care. A systematic review was conducted in September 2014 in three indexed databases: CAPES periodicals, SciELO and MEDLINE. An article which described the construction of a logical model has been identified. After analysis of the logical model proposed and official documents of the Ministry of Health, were carried out some modifications in the component of prevention, care component, process (retired guidance on healthy habits and lifestyles and physical activity programming, were added Therapeutic hypertensive groups, continuing education for hypertensive on healthy eating and encouraging physical activity). In the control component, care component, process (if added stratification). It is expected that the logical model proposed will serve as a "model" for the ESF in order to cause changes arising from the products generated by actions. Specific changes in behavior, knowledge, skills, or performance level program participant, which may include improving quality of life.
\end{abstract}

Keywords: Logic Model. Hypertension. Family Health Strategy.

1 Docente da Universidade Ceuma

2 Docente da Universidade Federal do Maranhão 
Introdução

As transições demográficas, nutricionais e epidemiológicas, ocorridas no século $X X$, resultaram em significativa diminuição das taxas de fecundidade e natalidade e no aumento progressivo da expectativa de vida e da proporção de idosos em relação aos demais grupos etários. Como consequência, tem-se observado uma maior participação das Doenças Crônicas não Transmissíveis (DCNT) no perfil de morbimortalidade da população e a diminuição da morbimortalidade por doenças infecciosas e parasitárias, apesar destas últimas ainda representarem um importante problema de saúde pública em diversos países. ${ }^{1}$

$\mathrm{Na}$ segunda metade do século $X X$ houve crescimento expressivo das DCNT, principalmente as cardiovasculares, neoplásicas, respiratórias e diabetes. Tais enfermidades são responsáveis por $63 \%$ de todas as 36 milhões de mortes ocorridas no mundo em $2008^{2}$ e por $80 \%$ daquelas ocorridas em países de média e baixa renda, no ano de 2005. $\mathrm{O}$ aumento epidêmico dessas doenças deveu-se às profundas mudanças sociais, econômicas, demográficas e culturais ocorridas no século passado. Se nada for feito para reduzir esse ônus, entre 2006 e 2015 serão perdidos US\$ 84 bilhões de produção econômica em 23 países de baixa e média renda, em decorrência de doenças cardiovasculares e diabetes. ${ }^{3}$

No cenário mundial, as DCNT representam, hoje, as principais causas de morte no Canadá e EUA, e também na América Latina e Caribe. ${ }^{4}$ No Brasil, em 2007, 72,0\% dos óbitos ocorridos no país foram decorrentes de DCNTs, sendo $31,3 \%$ de doenças do aparelho circulatório, 16,3\% câncer,
$5, \%$ diabetes e 5,8\% devido a doença respiratória crônica. ${ }^{5}$ Carnelosso et al. ${ }^{6}$ acrescentam que a expansão acelerada das doenças crônicas, como fato incontestável no mundo globalizado, constitui-se um desafio para as autoridades sanitárias e profissionais de saúde no desenvolvimento de políticas públicas capazes de conter essa epidemia que se pronuncia para um futuro muito próximo, devendo responder, mundialmente, por $80 \%$ da carga de doenças.

Considerando a gravidade do problema a Organização Mundial da Saúde (OMS) aprovou, em 2000, uma Resolução endossando a Estratégia Global para Prevenção e Controle de Doenças Não Transmissíveis com ênfase nos países em desenvolvimento. Duas Estratégias Globais merecem destaque. No ano de 2003, foi estabelecida a Convenção Quadro para o Controle do Tabaco, o primeiro tratado internacional da história sobre saúde pública, firmando um compromisso internacional para a adoção de medidas de restrição ao consumo de cigarros e outros produtos derivados do tabaco. ${ }^{3}$

Em 2004, foi aprovada a Estratégia Global para Alimentação Saudável, Atividade Física e Saúde, com quatro objetivos principais: a) reduzir os fatores de risco para as DCNT decorrentes da alimentação inadequada e inatividade física; b) aumentar a atenção e o conhecimento a respeito de alimentação e atividade física; c) encorajar o desenvolvimento, fortalecimento e implantação de políticas e planos de ação em nível global, regional, nacional e comunitário, incluindo a sociedade civil, o setor privado e a mídia; d) e monitorar dados científicos e influências-chave na alimentação e 
atividade física, com apoio para pesquisa e fortalecimento dos recursos humanos necessários. $^{7}$

Numa abordagem mais ampla, Rouquayrol e Almeida ${ }^{8}$ referem como fazendo parte das DCNTs as doenças cardíacas e as cerebrovasculares, os cânceres, a hipertensão, o diabetes, as doenças autoimunes, entre outras. Frise-se que as doenças cardiovasculares, apesar de sua diminuição em termos proporcionais são a principal causa de morte no Brasil. Dados mais recentes expressam que a mortalidade por doença cardíaca hipertensiva, no Brasil, cresceu 11\%, fazendo aumentar para $13 \%$ o total de mortes atribuíveis às doenças cardiovasculares em 2007, em comparação a $30 \%$ para doença cardíaca isquêmica e $32 \%$ para doença cerebrovascular. ${ }^{9}$

$\mathrm{Na}$ concepção de Mendes $^{10}$ as doenças crônicas, em particular a hipertensão e o diabetes, requerem monitoramento e ações que possam contribuir com o manejo adequado dessas patologias. Para o autor, essas doenças constituem, portanto, o grande desafio do século $\mathrm{XXI}$, pois representam uma grande sobrecarga no sistema de saúde que terá que se organizar para atender a esta demanda crescente.

De acordo com a VI Diretrizes Brasileiras da Hipertensão Arterial $(\mathrm{DBH}-\mathrm{VI})$ entre os fatores de risco para mortalidade, a Hipertensão Arterial é responsável por $40 \%$ das mortes por acidente vascular cerebral e $25 \%$ daquelas por doença coronariana. No SUS, as doenças cardiovasculares são responsáveis por 1.150 .000 das internações/ano, com um custo aproximado de 475 milhões de reais, sendo que nestes números não estão inclusos os gastos com procedimentos de alta complexidade. ${ }^{11}$
A hipertensão arterial sistêmica (HAS) é uma condição clínica multifatorial caracterizada por níveis elevados e sustentados de pressão arterial (PA). Associa-se frequentemente a alterações funcionais e/ou estruturais de órgãosalvo (coração, encéfalo, rins e vasos sanguíneos) e a alterações metabólicas, com consequente aumento de risco de eventos cardiovasculares fatais e não fatais. ${ }^{11}$

A HAS tem alta prevalência e baixas taxas de controle tornando-se um dos principais fatores de risco (FR) modificáveis e um dos mais importantes problemas de saúde pública. A mortalidade por doença cardiovascular (DCV) tem aumentado progressivamente com a elevação da PA a partir de $115 / 75 \mathrm{mmHg}$ de forma linear, contínua e independente. Em 2001, cerca de 7,6 milhões de mortes no mundo foram atribuídas à elevação da PA (54\% por acidente vascular encefálico AVE e 47\% por doença isquêmica do coração - DIC), sendo a maioria em países de baixo e médio desenvolvimento econômico e mais da metade em indivíduos entre 45 e 69 anos. No Brasil, as DCVs têm sido a principal causa de morte. Em 2007 ocorreram 308.466 óbitos por doenças do aparelho circulatório. Entre 1990 a 2006, observou-se uma tendência lenta e constante de redução das taxas de mortalidade cardiovascular. ${ }^{11}$

Destaque-se que, no Brasil, a prevalência média de HAS autorreferida na população acima de 18 anos, segundo a Vigilância de Fatores de Risco e Proteção para Doenças Crônicas por Inquérito Telefônico (Vigitel), é de $22,7 \%$, sendo maior em mulheres $(25,4 \%)$ do que em homens (19,5\%). A frequência de HAS tornou-se mais comum com a idade, mais marcadamente para as mulheres, alcançando mais de $50 \%$ na faixa etária de 55 anos ou mais de idade. 
Entre as mulheres, destaca-se a associação inversa entre nível de escolaridade e diagnóstico da doença: enquanto $34,4 \%$ das mulheres com até 8 anos de escolaridade referiam diagnóstico de HAS, a mesma condição foi observada em apenas $14,2 \%$ das mulheres com 12 ou mais anos de escolaridade. Para os homens, o diagnóstico da doença foi menos frequente nos que estudaram de 9 a 11 anos. ${ }^{12}$

Assim considerado, verifica-se que a hipertensão arterial é um problema de saúde pública necessitando de intervenção. Desta forma, pretende-se com este estudo realizar uma pesquisa bibliográfica sobre o modelo lógico da atenção à hipertensão arterial desenvolvidos pelas Equipes de Saúde da Família. Visto que o Modelo Lógico é uma proposta para organizar as ações componentes de um programa de forma articulada aos resultados esperados, apresentam-se também as hipóteses e as ideias que dão sentido à intervenção. Considerado um instrumento para explicitar a teoria do programa, a aplicação do modelo lógico resulta em processo que facilita planejar e comunicar, $o$ que se pretende com o programa e qual o seu funcionamento esperado.

Para tanto é necessário articular uma explícita descrição das ideias, hipóteses e expectativas que constituem a estrutura do programa e o seu funcionamento esperado. Em muitos casos, a teoria não é explicitada de forma detalhada nos documentos oficiais, dificultando uma análise adequada.

Assim, o modelo lógico serve como um organizador para desenhar avaliação e medidas de desempenho, focalizando nos elementos constitutivos do Programa e identificando quais questões de avaliação devem ser colocadas e quais medidas de desempenho são relevantes. $^{13}$

Sob esses fundamentos, espera-se que o modelo lógico sirva de parâmetro, fonte de pesquisa para as Equipes de Saúde da Família, a fim de que ocorra uma compreensão metodológica dos objetivos do programa de hipertensão com intuito de reduzir a mortalidade cardiovascular.

\section{Objetivo}

Realizar uma revisão sistemática sobre o modelo lógico da atenção à hipertensão arterial na atenção básica.

\section{Material e Método}

Trata-se de uma revisão sistemática, que inclui a análise de pesquisas relevantes que dão suporte para a tomada de decisão e a melhoria da prática clínica, possibilitando a síntese do estado do conhecimento de um determinado assunto, além de apontar lacunas do conhecimento que precisam ser preenchidas com a realização de novos estudos. Este método de pesquisa permite a síntese de múltiplos estudos publicados e possibilita conclusões gerais a respeito de uma particular área de estudo. $^{14}$

Para sua elaboração as seguintes etapas foram percorridas: a) estabelecimento da hipótese e objetivos da revisão sistemática; b) estabelecimento de critérios de inclusão e exclusão de artigos para a seleção da amostra; c) definição das informações a serem extraídas dos artigos selecionados e análise dos resultados. 
Formulou-se a seguinte questão norteadora para guiar a revisão sistemática: Qual o modelo lógico da atenção à hipertensão arterial desenvolvida pelas equipes de Saúde da Família? Os critérios de inclusão utilizados para a seleção da amostra foram artigos completos, disponíveis eletronicamente, nos idiomas português, inglês ou espanhol com data da publicação a partir de 2009, uma vez que se buscou o modelo lógico da atenção à hipertensão arterial desenvolvida pelas equipes de Saúde da Família. Por sua vez, como critério de exclusão, os artigos repetidos foram retirados da análise do estudo.

O levantamento dos artigos foi realizado no mês de setembro de 2014 em três bases de dados indexadas: Periódicos da Coordenação de Aperfeiçoamento de Pessoal de Nível Superior (CAPES), Scientific Electronic Library Online (SciELO) e Medical Literature Analysis and Retrieval System Online (MEDLINE). Dessa forma, procurouse ampliar o âmbito da pesquisa, minimizando possíveis vieses nessa etapa do processo de elaboração da revisão sistemática.

Para o levantamento dos artigos, buscou-se utilizar os descritores controlados da Biblioteca Virtual em Saúde, por meio dos Descritores em Ciências da Saúde (DeCS), como "hipertenso", "avaliação" e "qualidade"; "hipertenso", "assistência" e "qualidade". Em virtude das características específicas para 0 acesso de cada uma das bases de dados selecionadas, as estratégias utilizadas para a busca dos artigos foram adaptadas para cada uma, tendo sempre como eixo norteador a questão previamente elaborada e os critérios de inclusão.

\section{Resultados}

De acordo com a revisão sistemática foram localizados 10 artigos completos na base de dados dos periódicos CAPES, 08 artigos MEDLINE, 07 artigos no SciELO, todos em língua portuguesa $\mathrm{e}$ publicados em periódicos nacionais. Após a exclusão de 10 artigos que estavam disponíveis em mais de uma base de dados, foi realizada a leitura dos resumos e verificou-se que apenas 7 artigos se enquadravam nos critérios de inclusão e respondiam a questão norteadora, sendo este o número de artigos que compôs a amostra (quadro 1). Segundo Costa et al. ${ }^{15}$ :

a) Para a construção do modelo lógico, foram inicialmente identificados os problemas (ações de controle da hipertensão pouco efetivas, elevada prevalência de hipertensão e elevada morbimortalidade por doenças cardiovasculares). Após essa etapa, apontou-se a intervenção a ser avaliada (reorganização da atenção à hipertensão arterial, tendo como eixo a atenção básica), foram identificados os componentes da intervenção (ações de prevenção, de controle e o uso dos sistemas de informação) e subcomponentes (as ações de controle foram divididas em: diagnóstico, vinculação, tratamento e sistema de referência). Para cada componente e/ou subcomponente, foram especificados a estrutura necessária (recursos humanos e materiais) e o processo adequado (ações a serem realizadas), visando a resultados a curto e médio prazo que levariam a longo prazo à redução da morbimortalidade por hipertensão arterial e suas complicações cardiovasculares.

Através da revisão sistemática descrita acima foi identificado um artigo que descreveu a construção de 
Quadro 1 - Artigos localizados, de acordo com a base de dados indexada e ano de publicação.

\begin{tabular}{|c|c|c|c|}
\hline $\begin{array}{l}\text { BASE DE } \\
\text { DADOS }\end{array}$ & TÍTULOS & $\begin{array}{c}\text { ANO DE } \\
\text { PUBLICAÇÃO }\end{array}$ & AUTOR \\
\hline SciELO & $\begin{array}{l}\text { Desenvolvimento da promoção } \\
\text { da saúde no Brasil nos } 2009 \\
\text { últimos vinte anos (1988-2008). }\end{array}$ & 2009 & $\begin{array}{c}\text { Buss e } \\
\text { Carvalho }\end{array}$ \\
\hline MEDLINE & $\begin{array}{l}\text { Preços e disponibilidade de } \\
\text { medicamentos no Programa } \\
\text { Farmácia Popular do Brasil }\end{array}$ & 2010 & Pinto et al. ${ }^{18}$ \\
\hline $\begin{array}{l}\text { Periódicos } \\
\text { CAPES }\end{array}$ & $\begin{array}{l}\text { Reconhecimento, tratamento e } \\
\text { controle da hipertensão arterial: } \\
\text { Estudo Pró-Saúde, Brasil }\end{array}$ & 2010 & Nogueira et al. ${ }^{19}$ \\
\hline SciELO & $\begin{array}{l}\text { Avaliação da implantação da } \\
\text { atenção à hipertensão arterial } \\
\text { pelas equipes de Saúde da } \\
\text { Família do município do Recife } \\
\text { (PE, Brasil). }\end{array}$ & 2011 & Costa et al. ${ }^{15}$ \\
\hline $\begin{array}{l}\text { Periódicos } \\
\text { CAPES }\end{array}$ & $\begin{array}{l}\text { A qualidade do cuidado ofertado } \\
\text { aos hipertensos na Atenção } \\
\text { Primária à Saúde e sua } \\
\text { efetividade na redução de } \\
\text { internações por causas } \\
\text { cardiovasculares em } \\
\text { Alegre/RS. }\end{array}$ & 2013 & Trindade $^{20}$ \\
\hline $\begin{array}{l}\text { Periódicos } \\
\text { CAPES }\end{array}$ & $\begin{array}{l}\text { Qualidade do atendimento nas } \\
\text { unidades de saúde da família no } \\
\text { município de Recife: a percepção } \\
\text { do usuários. }\end{array}$ & 2013 & Santiago et al. ${ }^{21}$ \\
\hline $\begin{array}{l}\text { Periódicos } \\
\text { CAPES }\end{array}$ & $\begin{array}{l}\text { Do normativo a realidade do } \\
\text { sistema Único de Saúde: } \\
\text { revelando barreiras de acesso na } \\
\text { rede de cuidados assistenciais. }\end{array}$ & 2014 & Sousa et al. ${ }^{22}$ \\
\hline
\end{tabular}


um Modelo Lógico da atenção à hipertensão arterial desenvolvida pelas equipes de Saúde da Família (figura 1).

Após análise do modelo lógico proposto por Costa et al.15 e dos documentos oficiais do Ministério da Saúde, como manuais, portarias e as leis que tratam da atenção ao hipertenso, foram realizadas algumas modificações nos componentes de prevenção, de cuidado-processo (acrescentou-se o grupo de hipertensos com reuniões periódicas). No componente controle, componente do cuidado processo (acrescentou-se estratificação de risco para os hipertensos). Frise-se ainda que os componentes de intervenção e componentes do cuidado (estrutura, processo, resultado intermediário e resultado final), foram identificados por cores com intuito de melhorar a compreensão metodológica do modelo lógico (figura 2).

Figura 1 - Modelo lógico da atenção à hipertensão arterial desenvolvida pelas equipes de Saúde da Família ${ }^{15}$

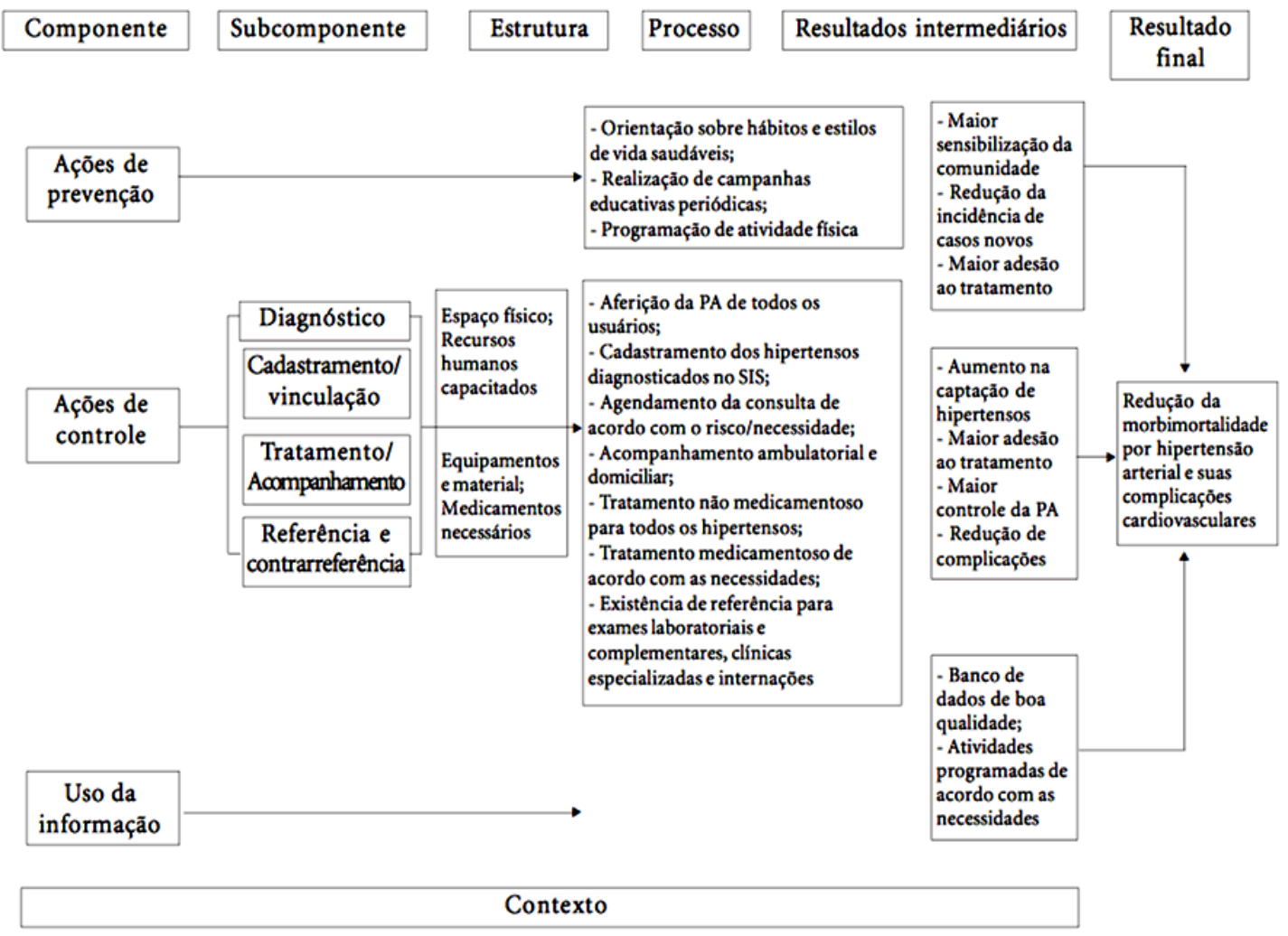


Figura 2 - Modelo Lógico da atenção à hipertensão arterial desenvolvida pelas equipes de Saúde da Família

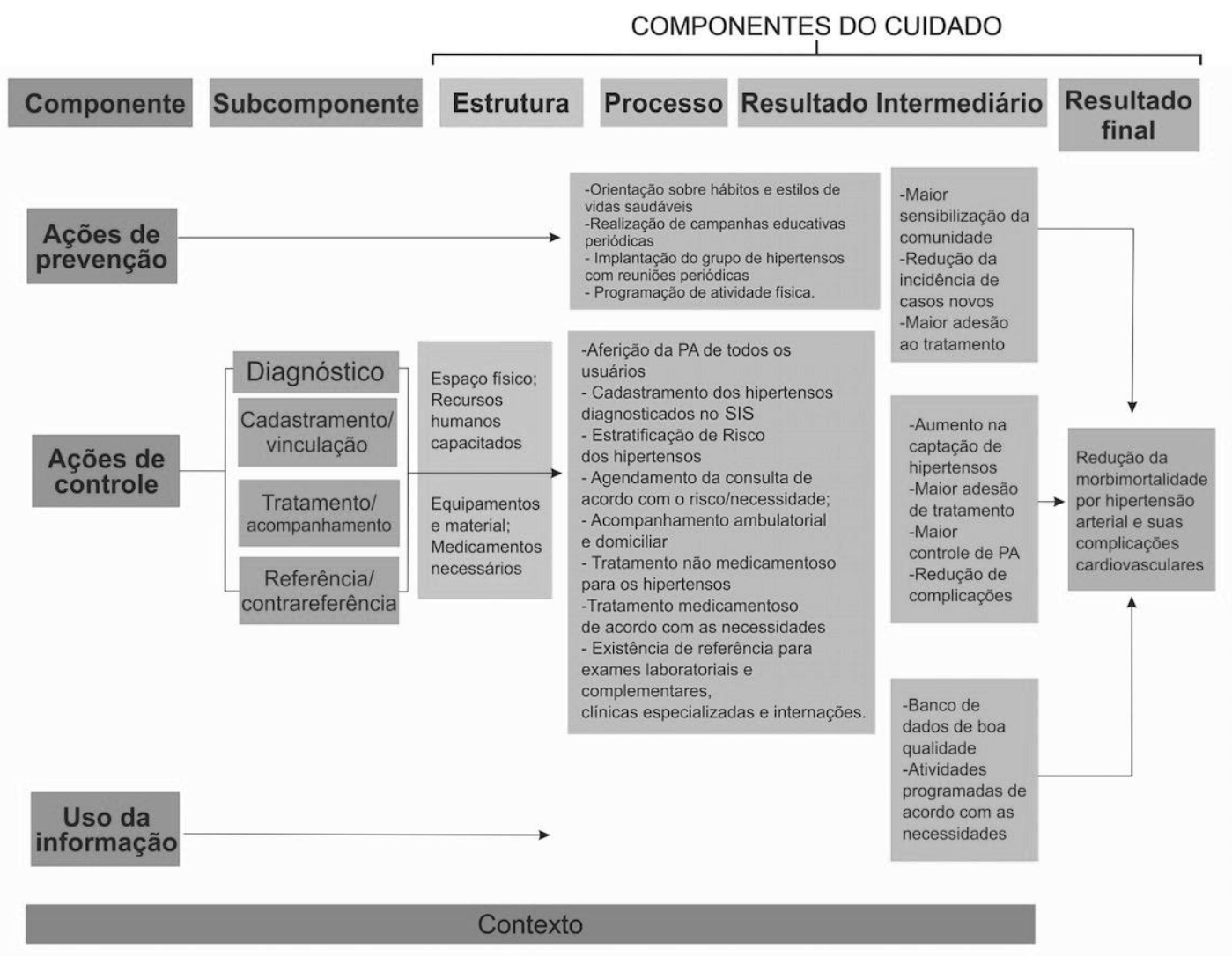

\section{Discussão}

O modelo lógico é uma proposta para organizar as ações, componentes de um programa, de forma articulada aos resultados esperados, apresentando também as hipóteses e as ideias que dão sentido à intervenção. Considerado um instrumento para explicitar a teoria do programa, a aplicação do modelo lógico resulta em um processo que facilita planejar $e$ comunicar o que se pretende com o programa e qual 0 seu funcionamento esperado. ${ }^{16}$

Em particular, pode ser utilizado como um instrumento para se proceder a avaliação ex-ante de programas, visando melhorar a consistência de sua formulação inicial. Alguns estudiosos da avaliação destacam a importância de partir-se da análise da teoria do programa para a identificação de deficiências do desenho que poderão interferir no seu desempenho. Aferir a qualidade da teoria significa, em síntese, verificar se o programa está bem desenhado e se apresenta um plano plausível para 0 alcance dos resultados esperados. Ou seja, se o desenho do programa contemplou a delimitação e a explicação do problema a ser enfrentado com a intervenção e se as ações planejadas têm como referências mudanças em causas do problema. $^{16}$ 


\section{Conclusão}

Após a realização deste trabalho espera-se que o modelo lógico sirva de "modelo" para as Equipes de Saúde da Família, a fim de que ocorram mudanças decorrentes dos produtos gerados pelas ações. Mudanças específicas no comportamento, conhecimento, habilidades, ou nível de desempenho do participante do programa, os quais poderão incluir melhoria da qualidade de vida, aumento da capacidade e/ou mudanças na arena política.

Desta forma, com a implantação e seguimento deste modelo lógico pretende-se:

a) reduzir a prevalência da obesidade dos hipertensos;

b) reduzir a prevalência de consumo de álcool dos hipertensos;

c) reduzir a prevalência de consumo de tabaco dos hipertensos;

d) aumentar a prevalência de atividade física dos hipertensos;

e) aumentar o consumo de frutas e hortaliças dos hipertensos;

f) reduzir o consumo diário de sal dos hipertensos;

g) monitorar a pressão arterial dos hipertensos mensalmente;

h) monitorar o índice de massa corporal dos hipertensos mensalmente e monitorar a adesão ao tratamento farmacológico.

\section{Agradecimentos}

Agradeço a todos que tornaram possível a realização deste trabalho, aqueles que contribuíram direta ou indiretamente para elaboração deste artigo meus sinceros agradecimentos.

\section{Conflito de Interesses}

O conflito de interesse da pesquisa está relacionado entre 0 pesquisador e os profissionais da atenção básica, pelo fato da pesquisa intervir diretamente no processo de trabalho das equipes de saúde da família.

\section{Contribuições:}

LBC Soares trabalhou na concepção e o delineamento ou a análise e interpretação dos dados e na redação do artigo.

IA Figueiredo trabalhou na versão a ser publicada.

\section{Referências}

1. Malta DC, Cezário AC, Moura L, Morais Neto OL, Silva JB Jr. A construção da vigilância e prevenção das doenças crônicas não transmissíveis no contexto do Sistema único de Saúde. Epidemiol Serv Saúde 2006; 15(3):47-65.

2. World Health Organization. Noncommunicable diseases country profiles 2011. Geneva; 2011.

3. Brasil. Ministério da Saúde. Secretaria de Vigilância em Saúde. Doenças crônicas não transmissíveis no Brasil: mortalidade, morbidade e fatores de risco. Brasília; 2008.

4. World Health Organization. Action plan for the global strategy for the prevention and control of noncommunicable diseases. Geneva; 2008. 
5. Freitas LRS, Garcia LP. Evolução da prevalência do diabetes e deste associado à hipertensão arterial no Brasil: análise da Pesquisa Nacional por Amostra de Domicílios, 1998, 2003 e 2008. Epidemiol Serv Saúde 2012; 21(1):7-19.

6. Carnelosso MS, Barbosa MA, Porto CC, Silva AS, Carvalho MM, Oliveira ALI. Prevalência de fatores de risco para doenças cardiovasculares na região Leste de Goiânia (GO). Ciên Saúde Coletiva 2010; 15(supl. 1):1073-80.

7. Organização Mundial da Saúde. Estratégia global em alimentação saudável, atividade física e saúde. In: Procedimentos dos 57aㅡ Assembleia Mundial da Saúde; 2004 maio 22; Geneva, Suiça. Geneva: WHO; 2004.

8. Rouquayrol MZ, Almeida NF. Epidemiologia e saúde. 6aㅡ ed. Rio de Janeiro: MEDSI; 2003.

9. Schmidt MI, Duncan BB, Silva GA, Menezes AM, Monteiro CA, Barreto SM, Chor D, Menezes PR. Doenças crônicas não transmissíveis no Brasil: carga e desafios atuais. Lancet, 2011; 61-74.

10. Mendes TAB. Prevalência de doenças crônicas e utilização dos serviços de saúde por idosos residentes no Município de São Paulo [tese]. São Paulo: Universidade de São Paulo; 2010.

11. Sociedade Brasileira De Cardiologia. VI Diretrizes Brasileira de Hipertensão. Arq Bras Cardiol 2010; 95(supl. 1): $1-51$.
12. Brasil. Ministério da Saúde. Secretaria de Vigilância em Saúde. Vigitel Brasil 2011: vigilância de fatores de risco e proteção para doenças crônicas por inquérito telefônico. Brasília: Ministério da Educação; 2012.

13. Nery M, Flaeschen M. O Ipea sobe o morro: os técnicos do Ipea foram ao Complexo do Alemão para avaliar impactos de intervenções urbanística. Desafios do Desenvolvimento 2010; 7(63):2-6.

14. Mendes S, Silveira CCCP, Galvão CM. Revisão integrativa: método de pesquisa para a incorporação de evidências na saúde e na enfermagem. Texto Contexto-Enferm 2008; 17(4): 758-64.

15. Costa JMBS, Silva MRF, Carvalho EF. de. Avaliação da implantação da atenção à hipertensão arterial pelas equipes de Saúde da Família do município do Recife (PE, Brasil). Ciên Saúde Coletiva 2011; 16(2):623-33.

16. Cassiolato M, Gueresi S. Como elaborar modelo lógico: roteiro para formular programase organizar avaliação. Brasília: Instituto de Pesquisa Econômica Aplicada; 2010.

17. Buss PM, Carvalho Al. Desenvolvimento da promoção da saúde no Brasil nos últimos vinte anos (1988-2008). Ciênc saúde coletiva 2009; 14(6):2305-16.

18. Pinto CBS, Miranda ES, Emmerick ICM, Costa NR, Castro CGSO. Preços e disponibilidade de medicamentos no Programa 
Farmácia Popular do Brasil. Rev saúde pública 2010; 44(4):6119.

19. Nogueira D, Faerstein E, Coeli CM, Chor D, Lopes CS, Werneck GL. Reconhecimento, tratamento e controle da hipertensão arterial: Estudo PróSaúde, Brasil. Rev Panam Salud Publica 2010; 27(2):1039.

20. Trindade TG. A qualidade do cuidado ofertado aos hipertensos na Atenção Primária à Saúde e sua efetividade na redução de internações por causas cardiovasculares em Porto Alegre/RS [tese]. Porto Alegre: Universidade Federal do Rio Grande do Sul; 2013.
21. Santiago RF, Mendes ACG, Miranda GMD, Duarte PO, Furtado BMASM, Souza WV. Título: Qualidade do atendimento nas Unidades de Saúde da Família no município de Recife: a percepção do usuários / Quality of care in the family healthcare units in the city of Recife: user perception. Ciênc saúde coletiva 2013; 18(1):35-44.

22. Sousa FOS, Medeiros KJ, Gurgel GD Jr, Albuquerque PC. Do normativo à realidade do Sistema Único de Saúde: revelando barreiras de acesso na rede de cuidados assistenciais. Ciênc saúde coletiva 2014; 19

23. (4): 1283-93. 